\title{
Measurement Placement in Distribution System State Estimation
}

\author{
Ravindra Singh, Student Member, IEEE, Bikash C. Pal, Senior Member, IEEE, and Richard B. Vinter, Fellow, IEEE
}

\begin{abstract}
This paper introduces a technique for meter placement for the purpose of improving the quality of voltage and angle estimates across a network. The proposed technique is based on the sequential improvement of a bivariate probability index governing relative errors in voltage and angle at each bus. The meter placement problem is simplified by transforming it into a probability bound reduction problem, with the help of the two sided-Chebyshev inequality. A straightforward solution technique is proposed for the latter problem, based on the consideration of $2-\sigma$ error ellipses. The benefits of the proposed technique are revealed by Monte Carlo simulations on a 95-bus UKGDS network model.
\end{abstract}

Index Terms-Bivariate Chebyshev bound, distribution management system, distribution system state estimation, error ellipse, measurement placement.

\section{INTRODUCTION}

$\mathbf{T}$ HE operation philosophy for power systems has been changing in the deregulated business environment. A key objective is now to improve operational efficiency through better utilization of network capacity. Another important goal is to accommodate a considerable portion of the distributed generation within an overall MW/MVAR transaction portfolio. This requires the monitoring and control of the network by means of a modern distribution management system (DMS) at substation level. State estimation should obviously be at the heart of the DMS technology. However, a large part of the system, particularly the distribution segment, continues to operate in an unmonitored fashion, adversely affecting the accuracy and quality of the state estimator and therefore its usefulness. This introduces bottlenecks in carrying out a range of substation and feeder automation tasks that rely on the quality of the state estimator.

Voltage, current and power flows are typically measured in a $33-\mathrm{kV}$ primary substation; virtually no monitoring is carried out further down at the secondary substation $(11 \mathrm{kV})$ level. The loads are not measured; instead they are modeled as "pseudo measurements" constructed from the historical and sample load profiles [2], [9]. Since pseudo measurements are high-variance estimates of the loads, the quality of the estimated voltages and angles at each bus is poor if the number of pseudo measurements

Manuscript received July 17, 2008; revised September 30, 2008. First published April 03, 2009; current version published April 22, 2009. This work was supported by EDF Energy Networks, U.K., under Grant EESC-P05821. Paper no. TPWRS-00579-2008.

The authors are with the Department of Electrical and Electronic Engineering, Imperial College, London, U.K. (e-mail: ravindra.singh@ic.ac.uk; b.pal@ic.ac.uk; r.vinter@ic.ac.uk).

Color versions of one or more of the figures in this paper are available online at http://ieeexplore.ieee.org.

Digital Object Identifier 10.1109/TPWRS.2009.2016457 is large. Indeed, estimation errors are often too high for effective network control; in this case it is necessary to deploy more real measurements. Economic considerations typically limit the number of measurement placements. Choices then need to be made regarding the locations, types and number of measurements. These issues have been addressed in the literature, at transmission level. But little attention has been paid to measurement placement at the distribution level. Research reported in the literature concerning measurement placement fall, broadly speaking, into two categories:

- the improvement of the network observability;

- the minimization of the errors in the estimates.

Monticelli and $\mathrm{Wu}[3]$ approached the problem from the network observability perspective. The idea is to add measurements until the network becomes fully observable. The linearized dc state estimation problem was subsequently solved iteratively with the addition of one measurement at a time. Gou and Abur [4] further refined this concept through a non-iterative technique that allowed simultaneous placement of several measurements. Very recently the benefits of placing some very accurate measurements (such as PMUs) to improve the observability [5] of the state estimation process has been demonstrated. The observability is tracked through the numerical conditioning of the linear measurement matrix. An optimal set of redundant measurements is also computed, relating to the loss of critical branches and measurements. The critical branches are identified from the system contingency analysis. All the measurements, however, are restricted to PMUs that give rise to a linear measurement matrix. The approach of placing the meters to achieve full observability is difficult to justify economically in distribution systems having very large number of nodes. A more cost effective approach is to combine the limited set of real measurements with a number of pseudo and virtual measurements.

Schweppe and Wildes [6] have suggested a meter placement technique that aims to reduce the variance of key estimated variables, such as the voltage and angle. Unlike the minimization of the variance of estimates in [6], Koglin [7] minimizes an index that is a weighted function of the variances of the quantity of interest. Their approach was later extended by Aam and others [8] and its robustness was improved by minimizing the sensitivity of the estimated error to the loss of redundant measurements.

All this work reported in the literature addresses the placement issues at the transmission system level. Concerning the distribution system, Li [9] demonstrated the impact of measurement location on the accuracy of the estimated states in a sample five-bus, four-feeder segments of RG\&E utility service area model. Baran and others [10] introduced a rule-based technique of meter placement. They were motivated by the fact 
that the accuracies of the states in individual locations are not equally important. Accurately estimating the load is not as important as getting a good estimate of the flow in the switching locations. The meters are assumed to be placed in the main switch and fuse locations first. The measurements are used for the switch and feeder monitoring and control functions. Additional measurements are then installed such that the total loads in each meter zone are similar in magnitude. The meters are also placed in the normal open points for the purpose of feeder switching and the feeder reconfigurations. The set of measurements is then reduced based on the accuracy index which is essentially the sum of the variances of the measurements. Shafiu and others [11] have applied a heuristic technique that identifies locations to place a certain number of voltage measurements to minimize the standard deviation in voltage estimates in non metered bus. The idea is to increase the distributed generation (DG) output without violating the voltage and flow constraints. A predetermined number of measurements is chosen arbitrarily. The method seeks to reduce errors in voltage magnitudes alone. But reducing errors in the estimation of angle estimates is also very important for the accurate computation of power flows and currents. Our research focuses on simultaneous reduction of the errors in both bus voltage magnitude and angle.

We adopt a probabilistic approach to meter placement, based on Monte Carlo simulations. The objective is to bring down the relative errors in the voltage and angle estimates, at all buses, below some predefined thresholds in more than $95 \%$ of the simulated cases. The idea is to identify measurement locations that reduce the "area" of the associated error ellipses. Details of this procedure appear in Section III. In Section II we give the results of simulations that reveal the effects on state estimation of varying the accuracy of the real and pseudo measurements, for no additional real measurements. In Section IV, a sequential meter placement technique is presented, based on reducing the area of 2- $\sigma$ error ellipse at candidate locations. The error ellipse is determined either from the errors in the voltage and angle at a bus or from the errors in real and reactive power flow in a line. The UKGDS network model is used to demonstrate the effectiveness of the method in Section V. Unlike many distribution systems, the U.K. distribution network is reasonably balanced. For this reason we have not considered phase wise computation; but the methodology is generic and may be employed also in this setting.

\section{State Estimation}

The weighted least square (WLS) estimator is commonly used for power system state estimation [1]. It can be shown that WLS gives consistent performance under Gaussian assumptions for known noise characteristics [first (mean) and second (variance) moments]. A brief overview of the WLS followed by its application on UKGDS model is provided in this section.

\section{A. WLS Estimator}

Let $\mathbf{x}$ denote the state vector, comprising voltage magnitudes and angles. The nonlinear measurement model relating the state vector and the measurement vector $(\mathbf{z})$ can taken to be

$$
\mathbf{z}=\mathbf{h}(\mathbf{x})+\mathbf{e}_{z}
$$

where $\mathbf{e}_{z} \sim \mathcal{N}\left(\mathbf{0}, \boldsymbol{R}_{z}\right)$ is zero mean Gaussian noise with measurement error covariance matrix $\boldsymbol{R}_{z}=\operatorname{diag}\left\{\sigma_{z 1}^{2}, \sigma_{z 2}^{2}, \ldots, \sigma_{z m}^{2}\right\} . \sigma_{z i}^{2}$ is the variance of the $i$ th measurement. $\mathbf{h}(\mathbf{x})$ is the vector of known nonlinear functions.

The WLS incorporates the above measurement model and minimizes the negative log-likelihood function in order to provide the optimal estimate of the state as follows:

$$
J=[\mathbf{z}-\mathbf{h}(\mathbf{x})]^{T} \boldsymbol{R}_{z}^{-1}[\mathbf{z}-\mathbf{h}(\mathbf{x})] .
$$

In calculating the minimand $\hat{\mathbf{x}}$, it is customary to employ a Newton scheme based on approximations to the first and second derivatives of $J$ as follows:

$$
\begin{aligned}
\nabla J(\hat{\mathbf{x}}) & =-2 \boldsymbol{H}^{T}(\hat{\mathbf{x}}) \boldsymbol{R}_{z}^{-1}[\mathbf{z}-\mathbf{h}(\hat{\mathbf{x}})] \\
\nabla^{2} J(\hat{\mathbf{x}}) & \approx 2 \boldsymbol{H}^{T}(\hat{\mathbf{x}}) \boldsymbol{R}_{z}^{-1} \boldsymbol{H}(\hat{\mathbf{x}})
\end{aligned}
$$

where

$$
\boldsymbol{H}(\hat{\mathbf{x}})=\left[\frac{\partial \mathbf{h}(\mathbf{x})}{\partial \mathbf{x}}\right]_{\mathbf{x}=\hat{\mathbf{x}}} \text { and } \hat{\mathbf{x}}=\lim _{k} \hat{\mathbf{x}}_{k}
$$

The iterates $\hat{\mathbf{x}}_{k}$ are calculated according to

$$
\begin{aligned}
\hat{\mathbf{x}}_{k+1}=\hat{\mathbf{x}}_{k}+\left(\boldsymbol{H}^{T}\left(\hat{\mathbf{x}}_{k}\right) \boldsymbol{R}_{z}^{-1}\right. & \left.\boldsymbol{H}\left(\hat{\mathbf{x}}_{k}\right)\right)^{-1} \\
& \times \boldsymbol{H}^{T}\left(\hat{\mathbf{x}}_{k}\right) \boldsymbol{R}_{z}^{-1}\left[\mathbf{z}-\mathbf{h}\left(\hat{\mathbf{x}}_{k}\right)\right] .
\end{aligned}
$$

We also employ an estimate of the state error covariance matrix $\hat{P}_{x}$ at the minimand $\hat{\mathrm{x}}[12]$ as follows:

$$
\hat{\boldsymbol{P}}_{x}=\left(\boldsymbol{H}^{T}(\hat{\mathbf{x}}) \boldsymbol{R}_{z}^{-1} \boldsymbol{H}(\hat{\mathbf{x}})\right)^{-1} .
$$

\section{B. Simulation Study}

To assess the performance of the state estimator in a distribution system, the WLS estimation technique was applied to a part of the U.K. Generic Distribution Network (UKGDS) model. The system considered comprises 95 buses, with two wind farms as sources of DG. Fig. 1 shows the schematic of the test system. The network and load data for UKGDS were obtained from [13]. For the state estimation, it was assumed that errors associated with the measurements are independent and identically distributed $(i . i . d$.). Three types of measurements were considered. The voltage and flow measurements at the 
main substation and the substations with DGs were taken as real measurements. Zero injections, with a very low variance $\left(10^{-8}\right)$, were modeled as the virtual measurements. The loads were modeled as the pseudo measurements. The cases corresponding to $1 \%$ and $3 \%$ errors in the real measurements and $20 \%$ and $50 \%$ errors in the pseudo measurements were considered for simulations. The error in the pseudo measurements was chosen on the basis of the uncertainty in the load estimates of various class of customers, like industrial, domestic and commercial. The loads of the industrial customer can be estimated fairly accurately. The load of the domestic customers is very difficult to estimate and will give rise to a large estimation error. The uncertainty in the commercial load estimates lies between the two. It was also taken into consideration that, for this choice, the maximum demand limits at various buses were not violated and linear approximation was therefore valid. The mean value for these measurements was obtained using the distribution system load flow. For a Gaussian distribution, a $\pm 3 \sigma$ deviation about the mean accounts for more than $99.73 \%$ of the area under the Gaussian curve. For a given $\%$ of maximum error about the mean $\mu_{z i}$, the standard deviation of the error was computed as follows:

$$
\sigma_{z i}=\frac{\mu_{z i} \times \% \text { error }}{3 \times 100}
$$

Justification of this formula is provided in the Appendix.

The relative errors in the voltages and angles were observed in 100 Monte Carlo simulations. Thresholds were specified for the relative errors in voltages and angles to assess the performance of the estimator under varying degrees of error in the measurements.

Figs. 2-5 show the plots of relative voltage and angle errors at different buses. It can be observed that, when the measurement errors are small, the relative estimation errors in the voltages and angles in more than $95 \%$ of simulation cases are below their respective thresholds (i.e., $1 \%$ for voltage error and $5 \%$ for angle error). It is evident from Fig. 3 that with the increase in errors in the pseudo measurements from $20 \%$ to $50 \%$, the voltage estimate errors do not violate their threshold in all the simulations, while the angle estimate errors violate their threshold in significant number of simulation cases. With increase in the errors in the real measurements from $1 \%$ to $3 \%$ both the errors in the voltage and the angle estimates violate the threshold limits (Fig. 4). This violation is significantly more for the voltage estimate errors than it is for the angle estimate errors. It can be understood from the simulation that the errors in the voltage estimates are highly influenced by the errors in the real measurements and less influenced by the errors in the pseudo measurements (loads). On the other hand the errors in angle estimates are influenced by both. In the case shown in Fig. 5, when the errors in both real and pseudo measurements are high, the errors in voltage and angle estimates significantly violate the limits. An efficient way to overcome this problem is to increase the number of real measurements, although it may not be economical to place large number of meters. Hence, a cost effective strategy for meter placement should take account of the following factors:

- location of meters;

- type of measurements;

- number of measurements.

In the next section, the theoretical framework and strategy for placing the meters is discussed.

\section{PROBLEM FORMULATION}

The problem of meter placement is to identify the effective locations and the number of real measurements, so that the following probability indices:

$$
\begin{aligned}
& p_{i}=\operatorname{Pr}\left\{\left|\frac{\hat{V}^{i}-V_{t}^{i}}{V_{t}^{i}}\right| \leq \boldsymbol{\epsilon}_{1},\left|\frac{\hat{\delta}^{i}-\delta_{t}^{i}}{\delta_{t}^{i}}\right| \leq \boldsymbol{\epsilon}_{2}\right\} \\
& \text { for } i=2, \ldots, n
\end{aligned}
$$

relating to the relative errors of the voltage and angle estimates throughout the network, are brought below their specified thresholds. Here,

$V_{t}^{i}, \delta_{t}^{i}=$ True value of voltage and angle at the $i$ th bus, respectively

$\hat{V}^{i}, \hat{\delta}^{i}=$ Estimated value of voltage and angle at the $i$ th bus, respectively.

Bus \#1 at the main substation is the reference bus with bus angle zero. The measurements at the main substations are generally available. So this bus is excluded from the problem formulation. We define

$$
\begin{aligned}
& \boldsymbol{\mu}^{i}=\left[\begin{array}{l}
\mu_{v}^{i} \\
\mu_{\delta}^{i}
\end{array}\right]=E\left[\begin{array}{l}
\hat{V}^{i} \\
\hat{\delta}^{i}
\end{array}\right]=\left[\begin{array}{c}
V_{t}^{i} \\
\delta_{t}^{i}
\end{array}\right] \\
& \boldsymbol{P}_{v \delta}^{i}=E\left[\left(\left[\begin{array}{c}
\hat{V}^{i} \\
\hat{\delta}^{i}
\end{array}\right]-\boldsymbol{\mu}^{i}\right)\left(\left[\begin{array}{c}
\hat{V}^{i} \\
\hat{\delta}^{i}
\end{array}\right]-\boldsymbol{\mu}^{i}\right)^{T}\right] \\
& \boldsymbol{\mu}_{\epsilon}^{i}=\left[\begin{array}{ll}
\epsilon_{1} V_{t}^{i} & \epsilon_{2}\left|\delta_{t}^{i}\right|
\end{array}\right]^{T} \text {. }
\end{aligned}
$$

Let $\hat{\mathbf{x}}_{v \delta}^{i}=\left[\begin{array}{ll}\hat{V}^{i} & \hat{\delta}^{i}\end{array}\right]^{T}$, and using (9) and (11), the probability index in (8) takes the following form:

$$
\begin{aligned}
p_{i} & =\operatorname{Pr}\left\{-\boldsymbol{\mu}_{\epsilon}^{i} \leq\left[\begin{array}{c}
\hat{V}^{i}-\mu_{v}^{i}, \\
\hat{\delta}^{i}-\mu_{\delta}^{i}
\end{array}\right] \leq \boldsymbol{\mu}_{\epsilon}^{i}\right\} \\
& =\operatorname{Pr}\left\{-\boldsymbol{\mu}_{\epsilon}^{i} \leq\left(\hat{\mathbf{x}}_{v \delta}^{i}-\boldsymbol{\mu}^{i}\right) \leq \boldsymbol{\mu}_{\epsilon}^{i}\right\} \\
& =1-\operatorname{Pr}\left\{\left(\hat{\mathbf{x}}_{v \delta}^{i}-\boldsymbol{\mu}^{i}\right)\right. \\
& \left.>\boldsymbol{\mu}_{\epsilon}^{i} \text { or }\left(\hat{\mathbf{x}}_{v \delta}^{i}-\boldsymbol{\mu}^{i}\right)<-\boldsymbol{\mu}_{\epsilon}^{i}\right\}
\end{aligned}
$$

The above probability can be increased by decreasing the following probability index:

$$
\begin{aligned}
p_{i}^{\prime} & =1-p_{i} \\
& =\operatorname{Pr}\left\{\left(\hat{\mathbf{x}}_{v \delta}^{i}-\boldsymbol{\mu}^{i}\right)>\boldsymbol{\mu}_{\epsilon}^{i} \text { or }\left(\hat{\mathbf{x}}_{v \delta}^{i}-\boldsymbol{\mu}^{i}\right)<-\boldsymbol{\mu}_{\epsilon}^{i}\right\} .
\end{aligned}
$$


Since, $\left(\boldsymbol{P}_{v \delta}^{i}\right)^{-1} \boldsymbol{\mu}_{\epsilon}^{i} \geq 0$, using [14, Theorem 10(b)], the tight bivariate two sided-Chebyshev bound for (13) in closed form can be expressed as

$$
\begin{aligned}
& \sup _{\hat{\mathbf{x}}_{v \delta}^{i} \sim\left(\boldsymbol{\mu}^{i}, \boldsymbol{P}_{v \delta}^{i}\right)} \operatorname{Pr}\left\{\left(\hat{\mathbf{x}}_{v \delta}^{i}-\boldsymbol{\mu}^{i}\right)\right. \\
> & \left.\boldsymbol{\mu}_{\epsilon}^{i} \text { or }\left(\hat{\mathbf{x}}_{v \delta}^{i}-\boldsymbol{\mu}^{i}\right)<-\boldsymbol{\mu}_{\epsilon}^{i}\right\} \\
= & \min \left(1, \frac{1}{\left(\boldsymbol{\mu}_{\epsilon}^{i}\right)^{T}\left(\boldsymbol{P}_{v \delta}^{i}\right)^{-1}\left(\boldsymbol{\mu}_{\epsilon}^{i}\right)}\right) .
\end{aligned}
$$

If $1 /\left(\boldsymbol{\mu}_{\epsilon}^{i}\right)^{T}\left(\boldsymbol{P}_{v \delta}^{i}\right)^{-1}\left(\boldsymbol{\mu}_{\epsilon}^{i}\right) \geq 1$, the above relation is obvious because the maximum probability of the left-hand side cannot exceed unity. For $1 /\left(\boldsymbol{\mu}_{\epsilon}^{i}\right)^{T}\left(\boldsymbol{P}_{v \delta}^{i}\right)^{-1}\left(\boldsymbol{\mu}_{\epsilon}^{i}\right)<1$, the bound in (14) can be written as

$$
\begin{aligned}
\operatorname{Pr}\left\{\left(\hat{\mathbf{x}}_{v \delta}^{i}-\boldsymbol{\mu}^{i}\right)>\boldsymbol{\mu}_{\epsilon}^{i} \text { or }\left(\hat{\mathbf{x}}_{v \delta}^{i}-\boldsymbol{\mu}^{i}\right)<-\boldsymbol{\mu}_{\epsilon}^{i}\right\} \\
\leq \frac{1}{\left(\boldsymbol{\mu}_{\epsilon}^{i}\right)^{T}\left(\boldsymbol{P}_{v \delta}^{i}\right)^{-1}\left(\boldsymbol{\mu}_{\epsilon}^{i}\right)}
\end{aligned}
$$

It will be shown later in Section V-A that the condition $1 /\left(\boldsymbol{\mu}_{\epsilon}^{i}\right)^{T}\left(\boldsymbol{P}_{v \delta}^{i}\right)^{-1}\left(\boldsymbol{\mu}_{\epsilon}^{i}\right)<1$ is satisfied for practically acceptable thresholds. Hence, the problem based on the reduction in Chebyshev bound results in feasible solution. In view of this, the probability index in (13) can be decreased by decreasing the right-hand side of (15). Since $\boldsymbol{\mu}_{\epsilon}^{i}$ is a constant vector $(2 \times 1)$, one way to decrease the right-hand side is to reduce the area of the error ellipse generated by the error covariance matrix $\boldsymbol{P}_{v \delta}^{i}$. A theoretical framework for obtaining the area of the error ellipse is now summarized.

\section{A. Geometric Interpretation of Error Covariance Matrix}

The equation of the $n$-dimensional "zero mean" error ellipsoid takes the form: $\mathbf{e}^{T} \boldsymbol{P}^{-1} \mathbf{e}=c$, where $\mathbf{e}$ is the error vector $(n \times 1), P$ is the error covariance matrix $(n \times n)$, and $c$ is a constant. Since $P^{-1}$ is a real symmetric matrix (a property of the error covariance matrix), there exists an orthogonal matrix $T$ such that $T^{-1} \boldsymbol{P}^{-1} T=T^{T} \boldsymbol{P}^{-1} T=\Lambda$. Here $\Lambda$ is a diagonal matrix with diagonal entries the eigenvalues $\left(\lambda_{1}, \ldots \lambda_{n}\right)$ of $\boldsymbol{P}^{-1}$ [15]. Now let $D$ be the diagonal matrix with diagonal entries $\left(\sqrt{c / \lambda_{1}}, \ldots, \sqrt{c / \lambda_{n}}\right)$ and consider the new vector variable $\mathbf{e}^{\prime}$ defined by the transformation $\mathbf{e}=\mathrm{TDe}^{\prime}$. Because $T$ is an orthogonal matrix (and therefore has unit determinant), the determinant of the transformation TD is $c^{n / 2} /\left(\lambda_{1} \ldots \lambda_{n}\right)^{1 / 2}=$ $c^{n / 2} / \operatorname{det}\left(P^{-1 / 2}\right)=c^{n / 2} \sqrt{\operatorname{det}(P)}$. It is easy to see that, in terms of the transformed vector variable, the equation of the ellipsoid is simply

$$
e_{1}^{\prime 2}+\cdots+e_{n}^{\prime 2}=1
$$

the unit $n$-sphere, whose volume we write $V$. The volume of the ellipsoid is just the volume $V$ scaled by the determinant of the transformation and is expressed as $V c^{n / 2} \sqrt{\operatorname{det}(P)}$. In the $(2 \times 2)$ case when $V=\pi$ (the area of unit disk), the area of error ellipse is $c \pi \sqrt{\operatorname{det} \boldsymbol{P}}$.

\section{Measurement Placement}

An obvious choice to improve the index in (8) is to place the voltage meters at the locations where $\sqrt{\operatorname{det} \boldsymbol{P}}$ is largest. The voltage measurements can efficiently bring down the relative errors in the voltage estimates below threshold, but in some cases the same may not be achieved for the angle estimates even with the help of a large number of voltage meters. The reasons for this are evident from Fig. 6(a) and (b). As shown in Fig. 6(a), at a given bus, the two axes of the error ellipse are not aligned in the direction of coordinate axes. This implies that the errors in voltage and angle estimates are correlated. In this case the error reduction in the voltage estimate consequently reduces the error in angle estimate and vice versa. The reduction of the error estimate in one variable with respect to the other depends upon the degree of correlation between the two. A stronger correlation implies that the error reduction in one variable significantly reduces the error in the other. In Fig. 6(b), the ellipse axes are in the direction of coordinate axes. This means the errors in voltage and angle estimates are uncorrelated and hence the reduction of one does not influence the other. For such cases other type of measurements are required. In this paper, we have used the line power flow measurements in addition to the voltage measurements to bring down the errors below their thresholds. The placement of the flow measurements is based on the reduction of the error ellipse derived from the real and reactive power flow in a line. The process for the location and number of these measurements is discussed next.

\section{A. Step-by-Step Process}

Step 1) Run WLS over a set of Monte Carlo simulations and observe the relative errors in voltages and angles in each simulation at all the buses.

Step 2) If in more than $95 \%$ of the cases the relative errors in the voltages and angles are below their specified thresholds, respectively (i.e., $1 \%$ for voltage $\left(\epsilon_{1}\right)$ and $5 \%$ for angle $\left(\epsilon_{2}\right)$ ), stop; else go to Step 3 .

Step 3) If only the relative errors in voltage estimates satisfy the criterion in Step 2, go to Step 6; else Step 4.

\section{Placement of Voltage Measurements}

Step 4) Take the mean of the state error covariance matrix over all the Monte Carlo simulations and extract the sub-matrices corresponding to the voltage and angle at each bus.

Step 5) At every bus compute the area of the error ellipse from the determinant of sub-matrix and identify the bus with the largest area and place the voltage measurement at this bus. If measurement is already present choose the bus with the next largest area. Go to Step 1.

Placement of Line Power Flow Measurements

Step 6) Compute the mean of error covariance matrix corresponding to the real and reactive power flow, in each line.

Step 7) For each line compute the area of the line flow error ellipse and place the flow measurement in the line with the largest area. If the measurement is already present choose the line with the next largest area. Go to Step 1. 


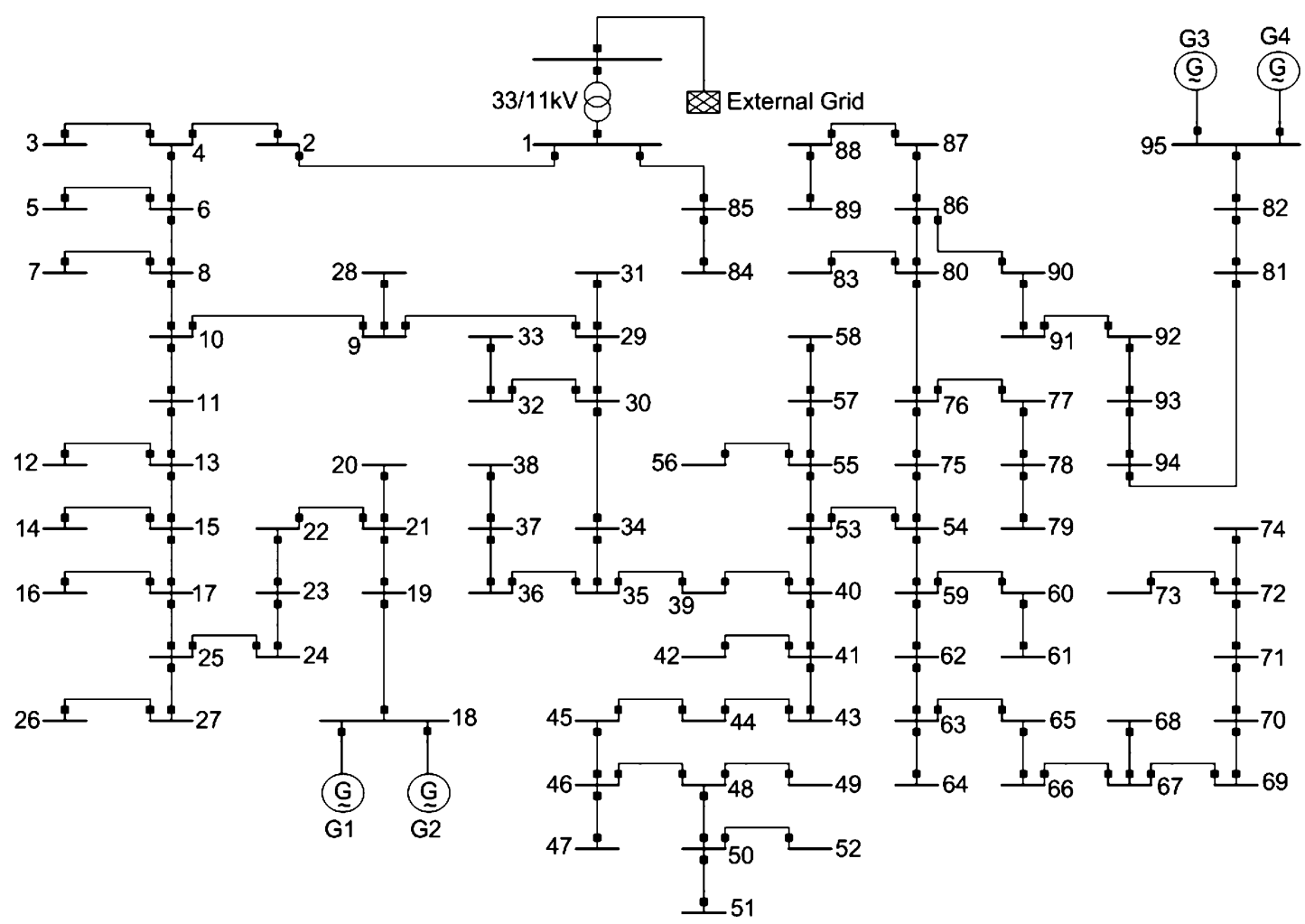

Fig. 1. UKGDS: 95-bus test system model.
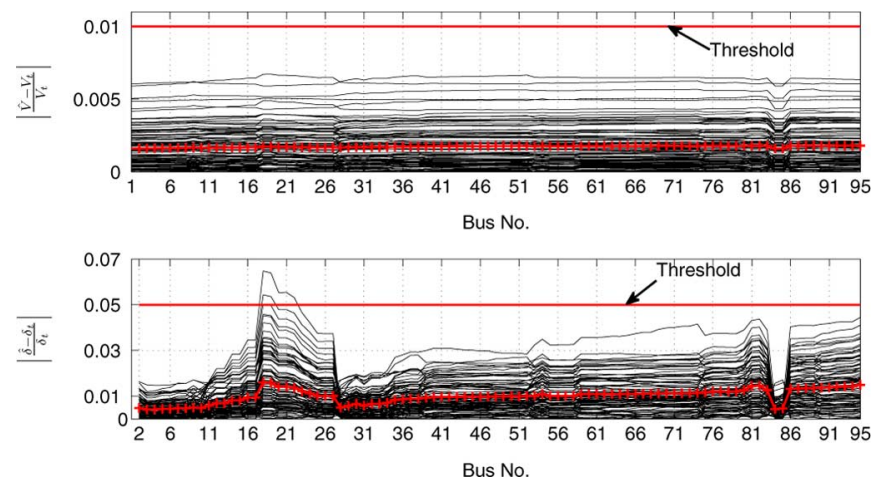

Fig. 2. Relative errors in voltage and angle estimates: with error in true measurements $=1 \%$, error in pseudo measurements $=20 \%$. Mean values of relative errors are represented by +++ .
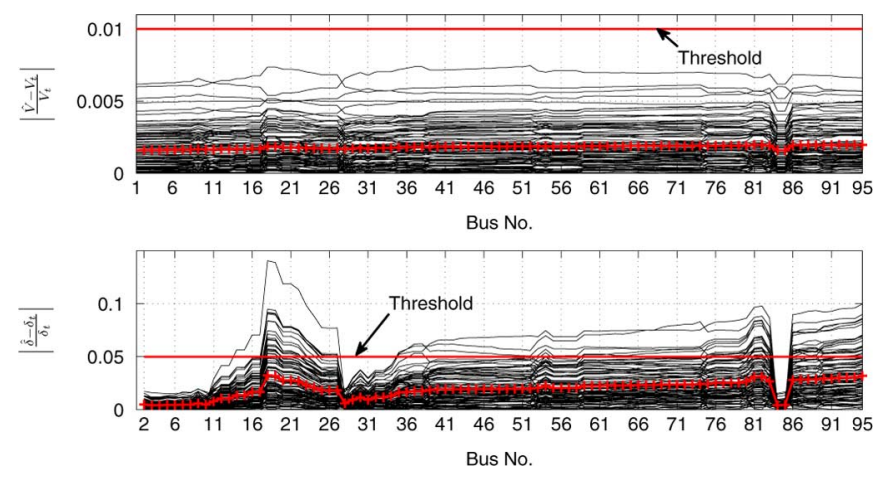

Fig. 3. Relative errors in voltage and angle estimates: with error in true measurements $=1 \%$, error in pseudo measurements $=50 \%$. Mean values of relative errors are represented by +++ .
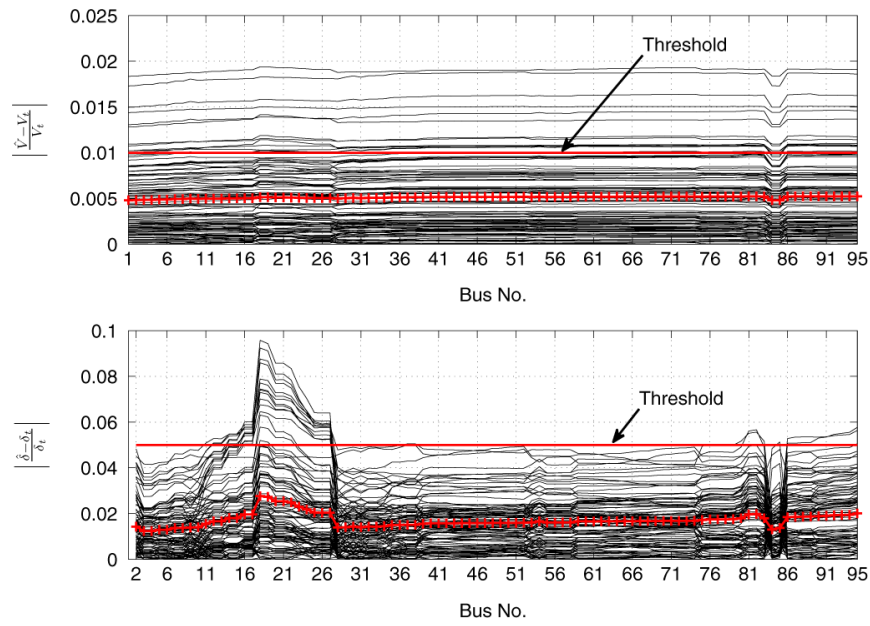

Fig. 4. Relative errors in voltage and angle estimates: with error in true measurements $=3 \%$, error in pseudo measurements $=20 \%$. Mean values of relative errors are represented by +++ .

The flow chart representation of the procedure is shown in Fig. 7.

\section{B. Optimality of the Proposed Scheme}

In this paper we address the meter placement strategy as a feasibility problem rather than an optimization one. The proposed method sequentially reduces the Chebyshev bound in order to improve the probability index in (8). The reduction in the bound stops when a pre-specified criterion is met (i.e., the relative errors in voltage and angle estimates are below their respective thresholds in more than $95 \%$ of the simulated cases). From the optimality perspective the results are suboptimal as the index 

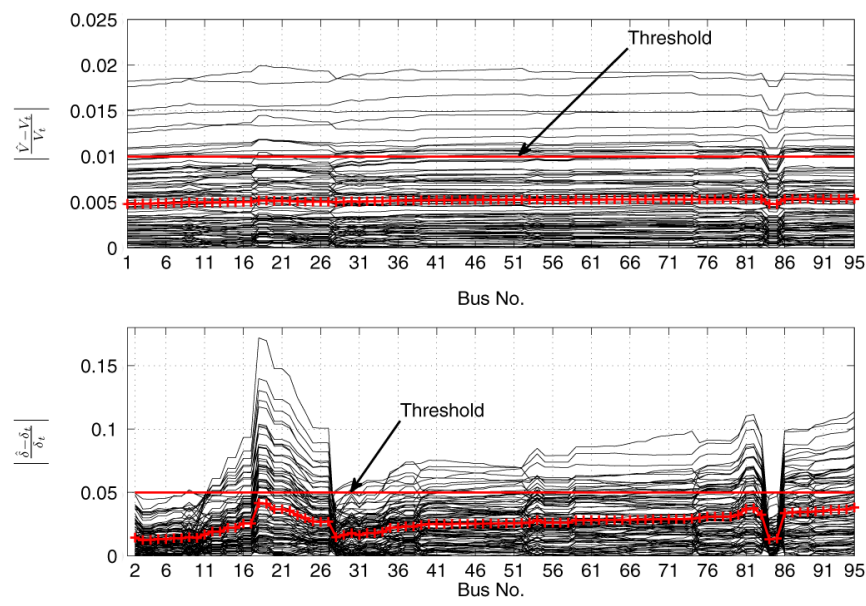

Fig. 5. Relative errors in voltage and angle estimates: with error in true measurements $=3 \%$, error in pseudo measurements $=50 \%$. Mean values of relative errors are represented by +++ .
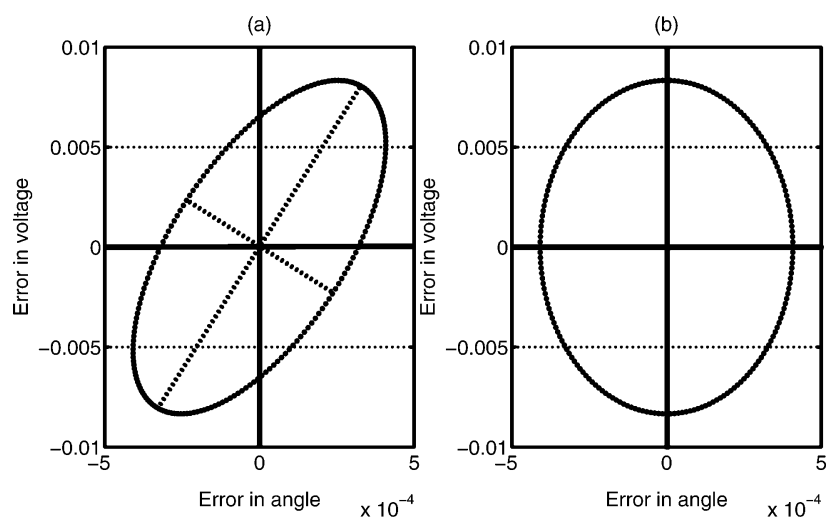

Fig. 6. Voltage and angle error ellipse: errors are (a) correlated, (b) uncorrelated.

in (8) can further be improved by increasing the number of real measurements. However, if the desired accuracy in the estimates is attained, improvements in state estimation resulting from further reductions of the probability indices is not economically justified.

\section{RESUlTS AND DISCUSSIONS}

The measurement placement technique discussed in the previous section was applied in the case of maximum measurement errors (i.e., $3 \%$ error in real measurements and 50\% errors in pseudo measurements). The plot of the relative errors in voltages and angles after applying the measurement placement technique is shown in Fig. 8. It was found that three additional voltage measurements at buses \#19, \#20, and \#21 and two power flow measurements in the lines \#15-17 and \#34-35 were needed to bring down the relative errors in voltages and angles below the specified thresholds in more than $95 \%$ of the simulated cases.

To assess the performance at each individual bus, the mean error ellipse at each bus over all the simulations was plotted. The error ellipse plots for some of the buses are shown in Fig. 9. The error ellipses corresponding to no additional measurements are shown in solid lines and those corresponding to the measurements after placement are displayed by dashed lines. It is clear from the plots that the proposed technique significantly reduces

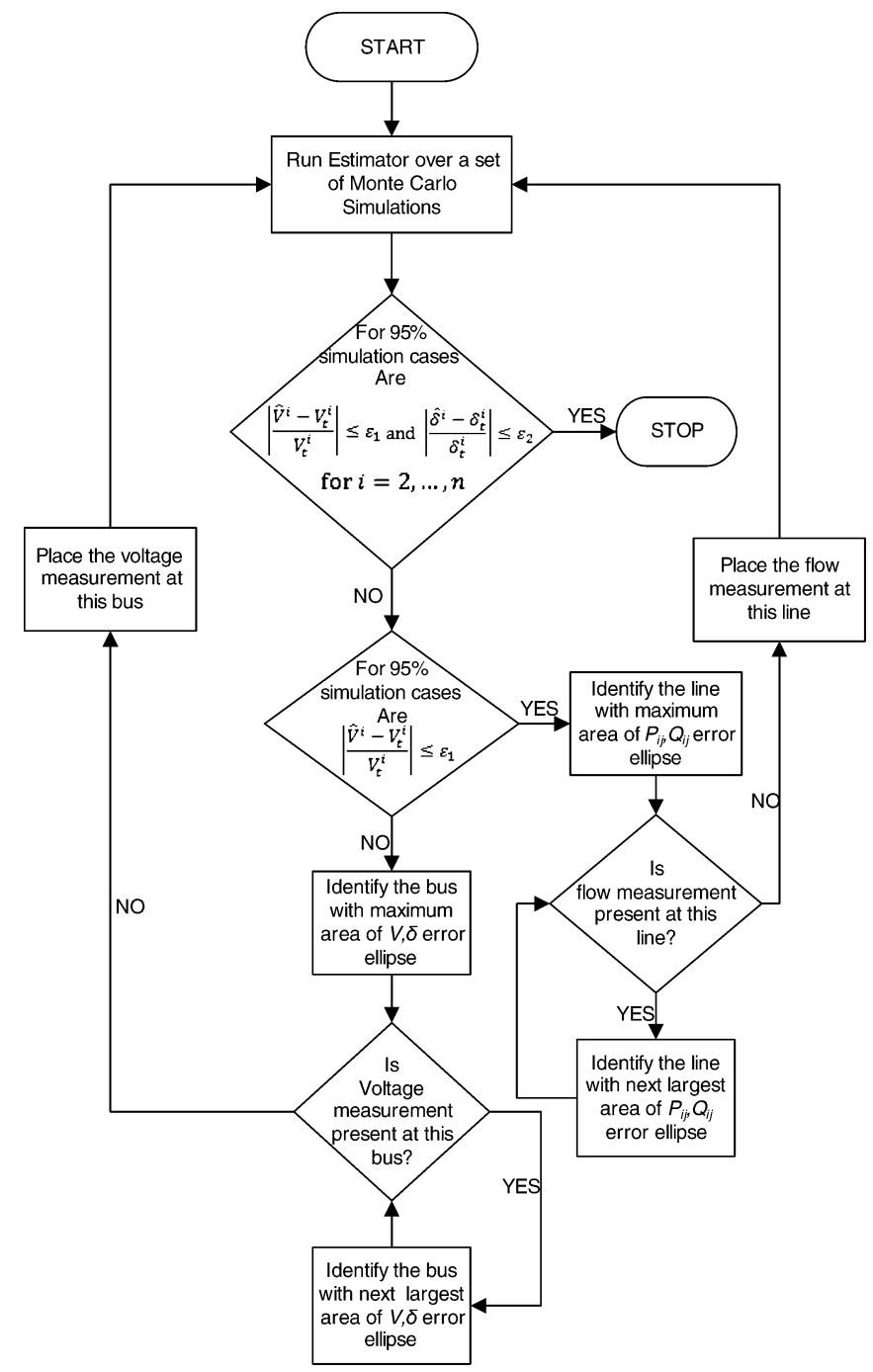

Fig. 7. Flow chart for measurement placement technique.
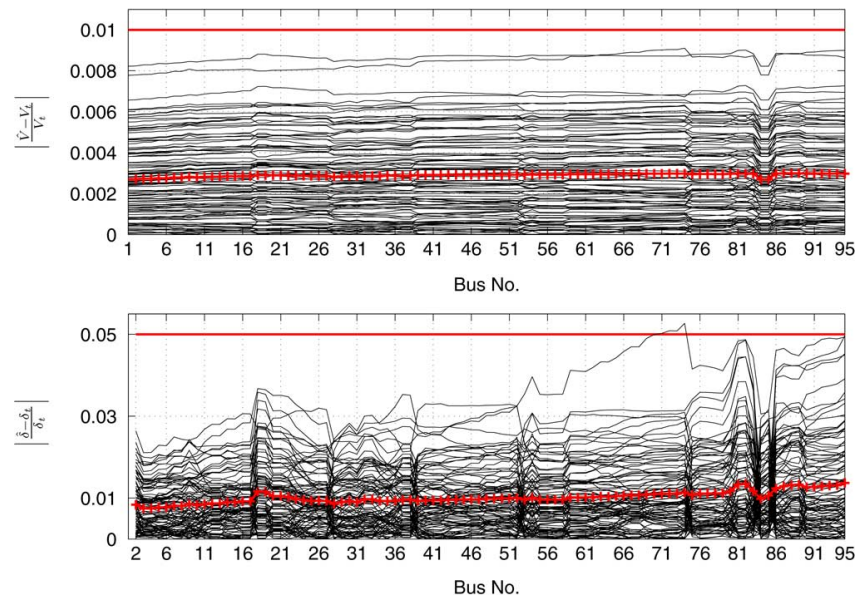

Fig. 8. Relative errors in voltage and angle estimates: with error in true measurements $=3 \%$, error in pseudo measurements $=50 \%$. Mean values of relative errors are represented by +++ .

the area of the ellipses at every bus in the network and hence improves the performance by reducing the error in the estimates. It should be noted that at bus \#1 there is no uncertainty along 

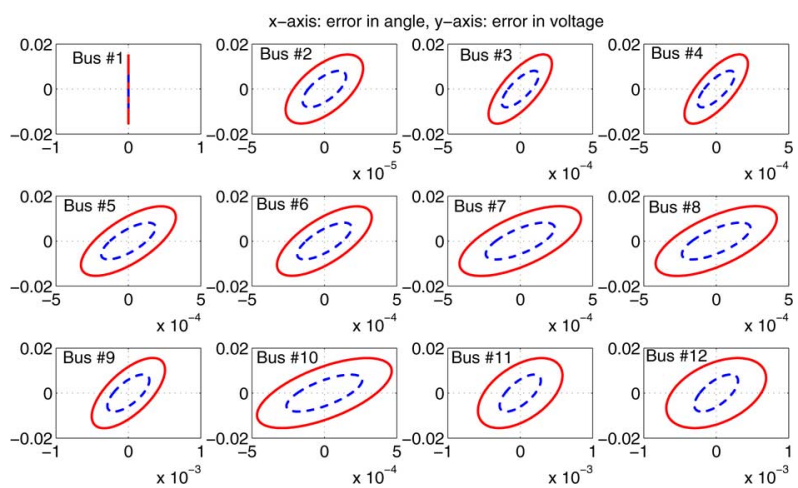

Fig. 9. Error ellipses at different buses. Solid ellipse: - - with no placement. Dashed ellipse: - - - with all five measurements placed.

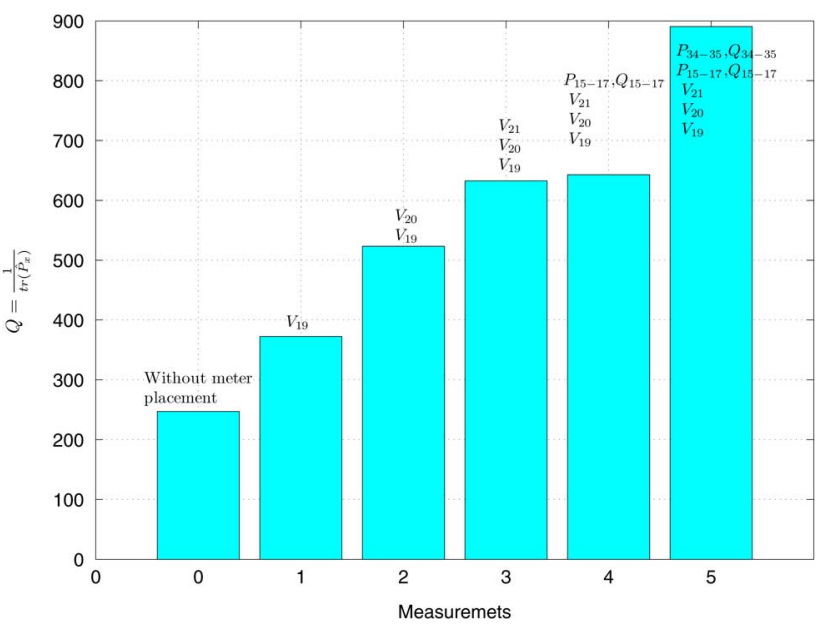

Fig. 10. Overall quality with measurement placement.

TABLE I

MINIMUM OF LOWER BOUND OF $p_{i}$, FOR $\epsilon_{1}=0.01, \epsilon_{2}=$ $0.05,\left(1-\max _{i}\left\{1 /\left(\boldsymbol{\mu}_{\epsilon}^{i}\right)^{T}\left(\boldsymbol{P}_{v \delta}^{i}\right)^{-1}\left(\boldsymbol{\mu}_{\epsilon}^{i}\right)\right\}\right)$

\begin{tabular}{|l||c|c|c|c|c|c|}
\hline \hline \multicolumn{1}{|c||}{} & \multicolumn{7}{c|}{ After Placement of Measurements } \\
\cline { 2 - 7 } & None & 1 & 2 & 3 & 4 & 5 \\
\hline \hline $\min \left\{p_{i}\right\}_{i=2}^{n} \geq$ & 0.839 & 0.873 & 0.894 & 0.904 & 0.917 & 0.949 \\
\hline \hline
\end{tabular}

$\mathrm{x}$-axis, because of the assumption of this bus as reference with bus angle zero.

In order to assess the overall performance, the quality of the estimates quantified in terms of the inverse of the trace of the mean error covariance matrix was evaluated with each additional meter placed. Fig. 10 shows the quality plot with no placement and with the measurements placed in various steps. It can be concluded that the placement technique significantly improves the overall quality of the estimates.

Table I shows the minimum value of lower bound of the probability index $p_{i}$ defined in (8). It is evident that with each measurement placed, the technique increases the probability index above the value specified in Table I. The index can attain a maximum value of unity in the limiting case. The proposed technique increases the probability index above 0.949 while satisfying the criteria on voltage and angle estimates. Although this

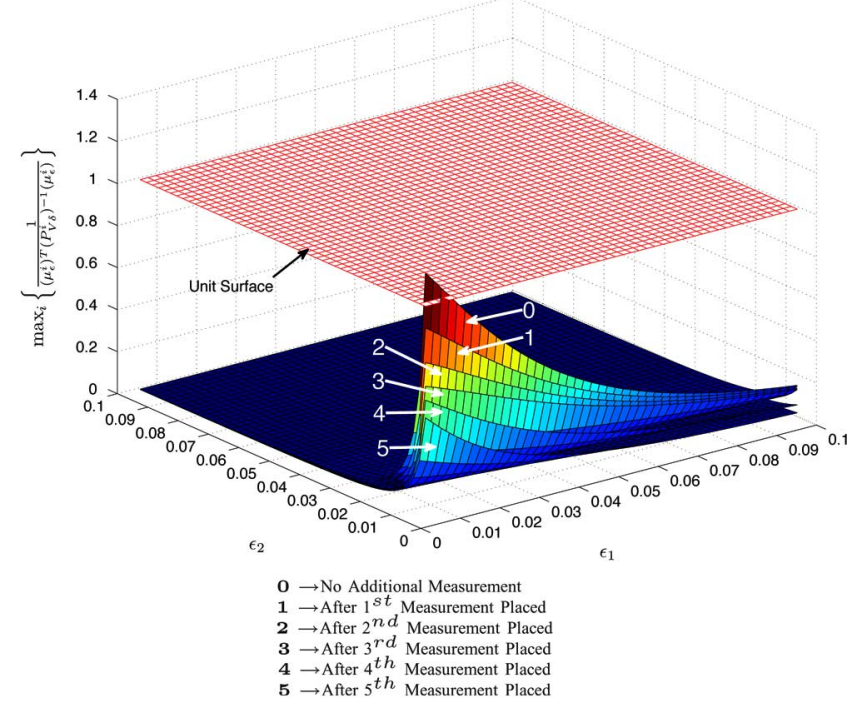

Fig. 11. Surfaces of the maximum Chebyshev bounds with measurement placement.

value is not optimal, it is not very far from the limiting value either.

\section{A. Comments on the Chebyshev Bound}

In the simulations, the thresholds, $\left(\epsilon_{1}, \epsilon_{2}\right)$, were varied from $0.05 \%$ to $10 \%$ and the maximum values of Chebyshev bound in (15) were evaluated. The surface plots of the maximum values of the Chebyshev bound, without and with each measurement placed are shown in Fig. 11. It is clear that for the chosen thresholds, the maximum bound is below unity. Hence, reduction in bound in (15) improves the probability index $p_{i}$ in (8). As the real measurements are introduced, the bounds decrease. It should be noted that, with no additional measurements the bound exceeds unity in a very few number of cases. These cases correspond to the selection of very small thresholds. In practice it is either difficult to achieve the relative errors below these thresholds due to the measurement constraints or sometimes impossible even with placement of real measurements at all possible locations. Furthermore, it may not be of any added significance to have such small thresholds for the benefit of the state estimation, as already observed in Section IV-B.

\section{CONCLUSION}

An analytical framework of meter placement is described in this paper. Geometrically the area of the error ellipse reflects the accuracy of the estimates. The area of the error ellipse in voltage-angle error plane for a particular node quantifies the influence that a measurement in that node can have on the overall accuracy of the state estimates. The proposed method seeks to find the location with largest area of the 2- $\sigma$ error ellipse as a potential location for meter placement. The procedure is sequential and stops when the desired level of accuracy in estimates is achieved. The advantage of the method is that it reduces the errors in both voltage and angles by exploiting the error correlations under a wide range of uncertainty in the pseudo measurements. The technique is simple and easy to implement. The performance evaluation of the technique on 95-bus UKGDS demonstrates the potential for practical implementation despite 
the fact that it produces feasible but not necessarily optimal solution. The performance of the technique needs further investigation in view of the possible presence of the leverage points in the network.

\section{APPENDIX \\ DERIVATION OF (7)}

Let $\epsilon_{m}^{i}$ denote the maximum \% error in the $i$ th measurement $z_{i} \sim \mathcal{N}\left(\mu_{z i}, \sigma_{z i}^{2}\right)$. Corresponding to the maximum \% error, we can define the following relation:

$$
\mu_{z i}-\mu_{z i} \frac{\epsilon_{m}^{i}}{100} \leq z_{i} \leq \mu_{z i}+\mu_{z i} \frac{\epsilon_{m}^{i}}{100} .
$$

The shifted and normalized form of the above relation can be expressed as

$$
-\frac{\mu_{z i}}{\sigma_{z i}} \frac{\epsilon_{m}^{i}}{100} \leq \frac{z_{i}-\mu_{z i}}{\sigma_{z i}} \leq \frac{\mu_{z i}}{\sigma_{z i}} \frac{\epsilon_{m}^{i}}{100}
$$

Since $z_{i}$ is a random variable, we can associate a probability with (18). Let this probability be $\alpha$. We can write

$$
\operatorname{Pr}\left(-\frac{\mu_{z i}}{\sigma_{z i}} \frac{\epsilon_{m}^{i}}{100} \leq \frac{z_{i}-\mu_{z i}}{\sigma_{z i}} \leq \frac{\mu_{z i}}{\sigma_{z i}} \frac{\epsilon_{m}^{i}}{100}\right)=\alpha .
$$

Using the cumulative distribution function for standard normal distribution, the above relation can be written as

$$
\Phi\left(\frac{\mu_{z i}}{\sigma_{z i}} \frac{\epsilon_{m}^{i}}{100}\right)-\Phi\left(-\frac{\mu_{z i}}{\sigma_{z i}} \frac{\epsilon_{m}^{i}}{100}\right)=\alpha .
$$

By the distribution symmetry, we have

$$
\begin{aligned}
& \Phi\left(\frac{\mu_{z i}}{\sigma_{z i}} \frac{\epsilon_{m}^{i}}{100}\right)-\left[1-\Phi\left(\frac{\mu_{z i}}{\sigma_{z i}} \frac{\epsilon_{m}^{i}}{100}\right)\right]=\alpha \\
& \Phi\left(\frac{\mu_{z i}}{\sigma_{z i}} \frac{\epsilon_{m}^{i}}{100}\right)=\frac{1+\alpha}{2} .
\end{aligned}
$$

Using the inverse transform in (22)

$$
\sigma_{z i}=\frac{\mu_{z i} \epsilon_{m}^{i}}{\Phi^{-1}\left(\frac{1+\alpha}{2}\right) \times 100} .
$$

For $\alpha=0.9973$, we have $\Phi^{-1}((1+0.9973) /(2))=3$. Substitution of this value in (23) results in (7).

\section{ACKNOWLEDGMENT}

The authors would like to thank P. D. Lang of EDF Energy Networks for his valuable suggestions and discussions.

\section{REFERENCES}

[1] A. Abur and A. G. Exposito, Power System State Estimation: Theory and Implementation. . New York: Marcel Dekker, 2004.

[2] A. K. Ghosh, D. L. Lubkeman, M. J. Downey, and R. H. Jones, "Distribution circuit state estimation using a probabilistic approach," IEEE Trans. Power Syst., vol. 12, no. 1, pp. 45-51, Feb. 1997.

[3] A. Monticelli and F. F. Wu, "Network observability: Identification of observable islands and measurement placement," IEEE Trans. Power App. Syst., vol. PAS-104, no. 5, pp. 1035-1041, May 1985.

[4] B. Gou and A. Abur, "An improved measurement placement algorithm for network observability," IEEE Trans. Power Syst., vol. 16, no. 4, pp. 819-824, Nov. 2001.

[5] C. Rakpenthai, S. Premrudeepreechacharn, S. Uatrongjit, and N. R. Watson, "An optimal PMU placement method against measurement loss and branch outage," IEEE Trans. Power Del., vol. 22, no. 1, pp. 101-107, Jan. 2007.

[6] F. C. Schweppe and J. Wildes, "Power system static-state estimation, part I, II, III," IEEE Trans. Power App. Syst., vol. PAS-89, no. 1, pp. $120-135$, Jan. 1970.

[7] H. J. Koglin, "Optimal measuring system for state estimation," in Proc. PSCC, Cambridge, U.K., Sep. 1975, Paper no. 2. 3/12.

[8] S. Aam, L. Holten, and O. Gjerde, "Design of the measurement system for state estimation in the Norwegian high voltage transmission network," IEEE Trans. Power App. Syst., vol. PAS-102, no. 12, pp. 3769-3777, Dec. 1983

[9] K. Li, "State estimation for power distribution system and measurement impacts," IEEE Trans. Power Syst., vol. 11, no. 2, pp. 911-916, May 1996.

[10] M. E. Baran, J. X. Zhu, and A. W. Kelly, "Meter placement for real time monitoring of distribution feeders," IEEE Trans. Power Syst., vol. 11, no. 1, pp. 332-337, Feb. 1996.

[11] A. Shafiu, N. Jenkins, and G. Strbac, "Measurement location for state estimation of distribution networks with generation," Proc. Inst. Elect. Eng., Gen., Transm., Distrib., vol. 152, no. 2, pp. 240-246, Mar. 2005.

[12] F. L. Lewis, Optimal Estimation.. New York: Wiley, 1986.

[13] United Kingdom Generic Distribution Network (UKGDS). [Online]. Available: http://monaco.eee.strath.ac.uk/ukgds.

[14] D. Bertsimas and I. Popescu, "Optimal inequalities in probability theory: A convex optimization approach," Sloan Working Paper No. 4083, Jun. 1999.

[15] G. Strang, Linear Algebra and Its Applications. . New York: Harcourt 1988.

Ravindra Singh (S'07) received the B.Tech. (with honors) degree from HBTI, Kanpur, India, and the M.Sc. (Engg.) degree from the Indian Institute of Science, Bangalore, India, in 2002, and 2004 respectively. Currently, he is pursing the Ph.D. degree at the Imperial College London, London, U.K.

His current research interests include distribution system state estimation.

Bikash C. Pal (M'00-SM'02) received the B.E.E. (with honors) degree from Jadavpur University, Calcutta, India, the M.E. degree from the Indian Institute of Science, Bangalore, India, and the Ph.D. degree from the Imperial College London, London, U.K., in 1990, 1992, and 1999, respectively, all in electrical engineering.

Currently, he is a Reader in the Department of Electrical and Electronic Engineering, Imperial College London. His current research interests include state estimation, power system dynamics, and flexible ac transmission system controllers.

Richard. B. Vinter (F'05) received the B.Sc. degree in engineering science from Oxford University, Oxford, U.K., and the Ph.D. degree in control engineering and the D.Sc. degree in mathematics, both from Cambridge University, Cambridge, U.K.

As a recipient of a Harkness Fellowship, he was a Postdoctoral Researcher at the Electronic Systems Laboratory, Massachusetts Institute of Technology, Cambridge, for two years. Since 1974, he has been on the academic staff of Imperial College, London, U.K., where he is now Professor of control theory. His research has centered on nonlinear control, optimization, differential games, tracking, and estimation. He has authored over 140 scientific papers and two textbooks. 